\title{
A Vision-Based Parking Lot Management System
}

\author{
Sheng-Fuu Lin, Yung-Yao Chen, and Sung-Chieh Liu
}

\begin{abstract}
Goals of parking lot management system include counting the number of parked vehicles, monitoring the changes of the parked vehicles over the time, and identifying the stalls available. To decrease the cost of the production, an integrated vision-based system is a good choice. In this paper, we propose a vision-based parking management system to manage an outdoor parking lot by four cameras set up at loft of buildings around it, sending information, including real-time display, to database of ITS center via internet. This system enables drivers to find parking spaces available or monitoring the parking lot where they parked their cars easily by wireless communication device. To increase accuracy, in the beginning, color manage is done to all input images, maintaining color consistency. Then, an adaptive parking lot background model is generated. The adequate color of each parking space is found out using statistical method in color image sequences captured by a camera, and foreground is extracted based on color information. The result will be further modified by shadow detection based on luminance analysis. Vision-based parking management system can manage large area by just several cameras. Adjusting position of the camera can easily make this system suitable for most cases. Besides, this system is endurable and is easy-installed because of its simple equipment.
\end{abstract}

\section{INTRODUCTION}

$\mathrm{P}$ arking lots are an important object class in many traffic and civilian applications. In the beginning, a parking lot management system just means a charge system which can count the number of parked cars and their elapsed time by paper cards. However, nowadays, an intelligent parking lot management system, would not simply manage the internal operation of a parking facility, but also should be designed to be compatible with a wide range of aspects that are intertwined with parking facilities. Undoubtedly, an intelligent parking lot management system plays an essential role in ITS (Intelligent Transportation Systems).

For internal operation, a complete parking lot management system must possess the functions of management, surveillance,

\footnotetext{
This work was supported in part by National Science Council, Taiwan, R.O.C., under Contract No. EX-91-E-FA06-4-4.

Sheng-Fuu Lin is with the Department of Electrical and Control Enginecring, National Chiao-Tung University, Taiwan, R.O.C.

(phone: 886-3-5712121 Ext. 54365; e-mail: sflin@mail.nctu.edu.tw).

Yung-Yao Chen is with the Department of Electrical and Control Engineering, National Chiao-Tung University, Taiwan, R.O.C.

(e-mail:shawn.ece93g@nctu.edu.tw).

Sung-Chieh Liu is with the Department of Electrical and Control Engineering, National Chiao-Tung University, Taiwan, R.O.C.

(e-mail:liujack0729@yahoo.com.tw).
}

and control. Goals of parking lot surveillance include counting the number of parked motor vehicles, monitoring the changes of the parked vehicles over the time, and identifying the location [1]. Advanced visual-based surveillance systems have to process videos coming from multiple cameras to detect the presence of mobile objects in the monitored scene [2]-[5]. As more methods of identifying simple movements become available, the importance of the contextual methods increases. Activities and movements are not only recognized at the moment of detection, but their interpretation or labeling is affected by the temporally extended context in which the events take place. Visual surveillance requires real-time interpretation or event classification of image sequences in order to automate the detection of predefined alarm situations in a given application domain [6]-[9]. Interesting examples of multisensor surveillance systems have been investigated and discussed in detail [10]-[12]. These systems have been applied successfully in visual security for both indoor and outdoor environments, such as banks, shopping malls, subway stations, and parking lots.

On the other hand, for external operation, a parking lot management system emphasizes the linking and integration of car parks and current traffic so that in-vehicle information system can be coordinated. In modern prosperous cities, there is a great need for advanced parking assistant systems to reduce the hustle for the drivers. Parking Guidance Information System (PGIS), also called as Advanced Public Transport System (APTS), can dynamically collect parking information, and provides real-time information service for drivers by the means of on-board guidance system or changeable message board installed on roadside, thus assisting drivers to conveniently and rapidly park [13]-[14]. PGIS was firstly used in Aachen, German in 1971, and was introduced to British in the late of 1970s. Then, PGIS was widely deployed in Japan and other Europe countries such as France. Moreover, the future parking management system is expected to provide advanced navigation service and optimized traffic management system [15]-[16].

Today, many drivers, especially less experienced drivers or out-of-towners, spend much time finding a parking space everyday. Moreover, this situation results in increased travel times and number of stops, unexpected delays, greater travels. Besides, nowadays, a surveillance system is quite common on the wayside or in the parking lot. If we have an algorithm to obtain more information such as the number and position of the unoccupied parking space from the camera in a simple way, it will help drivers to reduce the time of finding the parking lot 


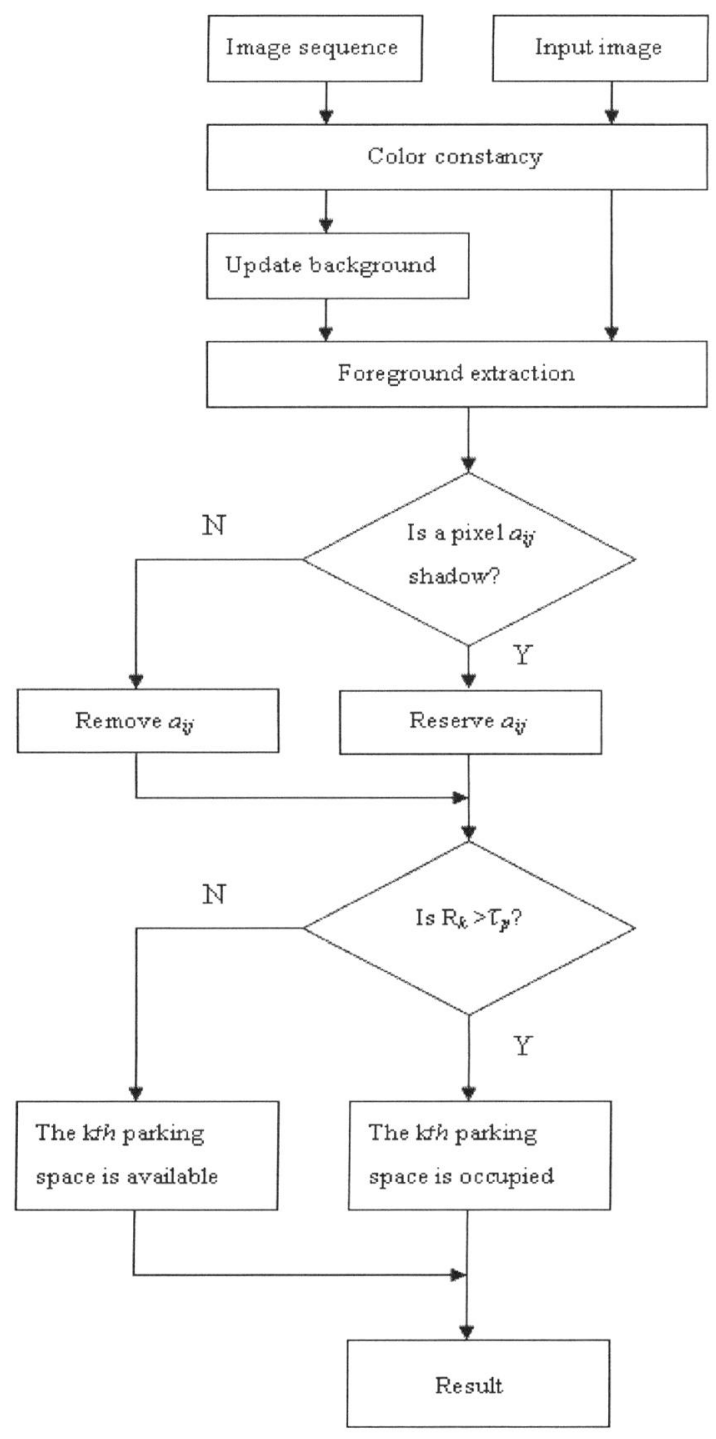

Fig. 1. General architecture of the entire system.

and to avoid the jams due to the driver's lowering speed and the meaningless search.

In this paper, we propose a vision-based method to deal with the parking lot management problem. In Sec. II, to maintain color stability, gray world assumption is preprocessed. Then, a method based on color can generate an adaptive background image and extract foreground image. At least, a shadow detection method will modify the foreground image and determine whether the stall is available. Fig. 1 shows the general architecture of the proposed vision-based parking lot management system.

\section{ALGORITHM}

\section{A. Color consistency}

Since our system is processing based on color information, it is necessary that a preprocessing procedure is needed to maintain color stability. However, images acquired from the
CCD camera may be affected due to different weather condition, thus the whole image looks somewhat bluish or reddish. In order to prevent color from shifting and make the system robust, the system have to do automatic white balancing to each incoming image. When implementing color balancing algorithm, certain assumptions are sometimes made about the general nature of the color components of images. There are a variety of white balancing algorithms. However, "gray world assumption" is used in this paper because of its simplicity. What gray world assumption states is that, given an image with sufficient amount of color variations, the average value of the red, green, and blue components of the image should average out to a common gray value. This assumption is in general valid since in any given real world scene, it is often the case where lots of different color variations are given. Since the variations in color are random and independent, it would be safe to say that given a large enough amount of samples, the average should tend to converge to the mean value, which is gray. The adjusted channel gains $R^{\prime}, G^{\prime}$, and $B^{\prime}$ can be obtained from

$$
\left\{\begin{array}{l}
Y_{a v g}=\left(R_{a v g}+G_{a v g}+B_{a v g}\right) / 3 \\
R^{\prime}=R \times \frac{Y_{a v g}}{R_{a v g}} \\
G^{\prime}=G \times \frac{Y_{a v g}}{G_{a v g}} \\
B^{\prime}=B \times \frac{Y_{a v g}}{B_{a v g}}
\end{array},\right.
$$

where the subscript avg represents average value. The result of using the gray world assumption is shown in Fig. 3(b).

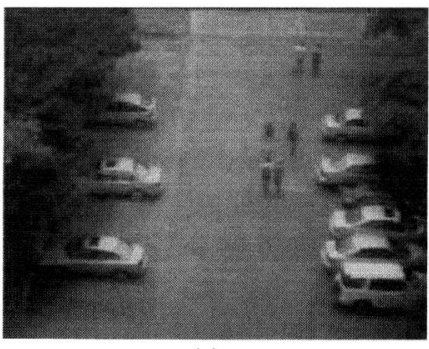

(a)

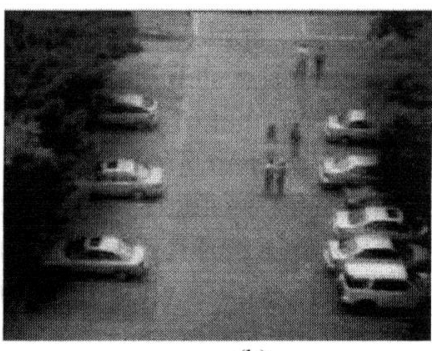

(b)
Fig. 2. (a) The original image. (b) Result of performing color consistency.

\section{B. Background generation}

In this section, an adaptive background will be generated for each parking space. Vehicles are considered as foreground and do foreground extraction to calculate the possibility of being occupied of each parking space. First, when a camera is set up, we will generate a parking lot model according to the image captured by the camera. In other words, because the position and the angle of the camera are fixed, we can define every parking space in advance. Fig. 3 shows the parking lot model. 
It seems to be bothersome to locate each space individually. However, a row of parking spaces are usually arranged side by side and horizontally. An expression for generating a parking lot model based on these assumptions may be written as

$$
B_{k}(x, y)=\left\{(x, y) \mid f\left(\theta_{n}, \theta_{p}, W_{k}, L_{k}\right)\right\} \text {. }
$$

The function $f\left(\theta_{n}, \theta_{p}, W_{k}, L_{k}\right)$ is given by

$$
f\left(\theta_{n}, \theta_{p}, W_{k}, L_{k}\right)=\left\{\begin{array}{l}
x_{0}-\left(i_{k} \sin \theta_{n}+j_{k} \cos \theta_{p}\right), i_{k}=1 \ldots W_{k} \\
y_{0}-\left(i_{k} \cos \theta_{n}+j_{k} \sin \theta_{p}\right), \mathrm{j}_{k}=1 \ldots L_{k}
\end{array},\right.
$$

where $B_{k}(x, y)$ are pixels belong to the $k$ th parking space, and $\left(x_{0}, y_{0}\right)$ is the starting point of $B_{k} \cdot \theta_{n}$ and $\theta_{p}$ represent the angle of the parking space, and $W_{k}, L_{k}$ are the width, length of the $k_{t h}$ parking space, respectively. In most cases, a row of parking spaces are aligned, so $\theta_{n}$ and $\theta_{p}$ are usually constants and it is easy to generate a parking lot by (2).

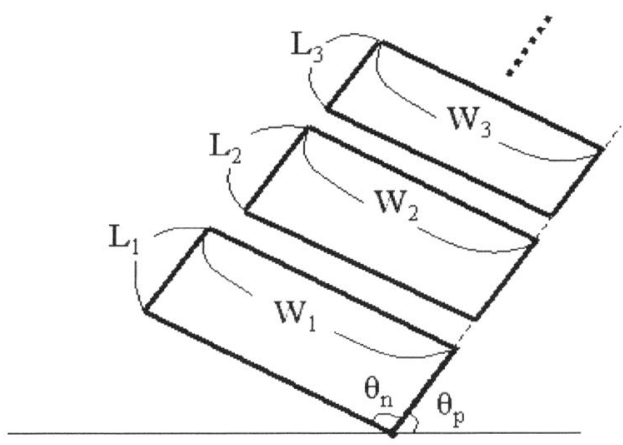

Fig. 3. Parking lot model

Next, it is imperative to find the fitting color to each parking space. It can be difficult to do parking lot management in an outdoor parking lot only by a constant background. These difficulties arise from shadows, changes of sunlight, and occlusion by other vehicles or pedestrians. The great variety of actual situations necessitates an adaptive background. In fact, because it is known that in most parking lots, the color of road is approximately the same as the color of parking space, determining the stalls available will be easier if the information about the road is known in advance. Therefore, in the system, an image which removes noise of pedestrians and vehicles on the road from image sequences can be generated by

$$
\hat{f}(x, y)=\operatorname{median}\left\{f_{n}(x, y)\right\}, n=1, \ldots, 60,
$$

where $f_{n}(x, y)$ is intensity (gray scale) value converted from RGB color space at coordinate $(x, y)$ in the input image at time $n$, and $\hat{f}$ is the output image. Since compared with the road, pedestrians and vehicles are relatively moving subjects, it is efficient to use median of image sequences to get the original color of the road. The result of using (4) is shown in Fig. 4(a), and the background image is shown in Fig. 4(b). The system can renew it at regular intervals. Then, in this image, deducing the color of each unoccupied parking space can be efficiently by using information of the predefined road area $S_{k}(x, y)$ which is near every parking space. Thus, we can get background image by coloring fitting colors to each parking space in the input image.

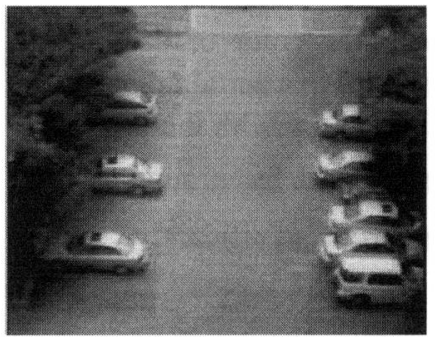

(a)

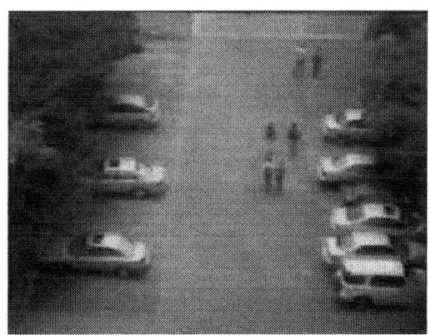

(c)

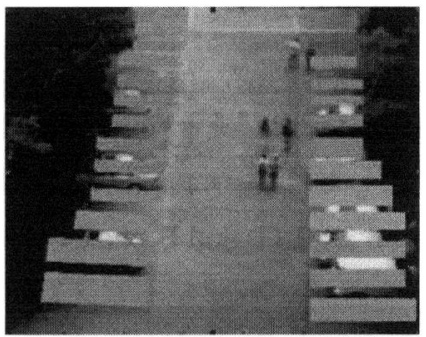

(b)
Fig. 4. (a) Image after (4): the noise of pedestrians would be removed, and the original color of the road is reserved. (b) The background image. (c) Comparing with the original image.

\section{Foreground extraction}

After generating the background image of every parking space, the vehicles in a color image can be defined when the RGB values of background pixels significantly differ. So, the foreground image can be extracted by following equation

$$
P(x, y)=\left\{\begin{array}{rl}
\text { if }\left|I_{R}(x, y)-M_{R}(x, y)\right| & <\sigma_{k R} \\
1, \text { and }\left|I_{G}(x, y)-M_{G}(x, y)\right| & <\sigma_{k G} \\
\text { and }\left|I_{B}(x, y)-M_{B}(x, y)\right| & <\sigma_{k B} \\
0, \text { otherwise }
\end{array} .\right.
$$

The value of $\left(\sigma_{k R}, \sigma_{k G}, \sigma_{k B}\right)$ will be obtained by

$$
\left\{\begin{array}{l}
\sigma_{k R}=\sqrt{\frac{1}{N_{S_{k}}} \sum_{(x, y) \in S_{k}}\left|I_{R}(x, y)-m_{k R}(x, y)\right|^{2}} \\
\sigma_{k G}=\sqrt{\frac{1}{N_{S_{k}}} \sum_{(x, y) \in S_{k}}\left|I_{G}(x, y)-m_{k G}(x, y)\right|^{2}}, \\
\sigma_{k B}=\sqrt{\frac{1}{N_{S_{k}}} \sum_{(x, y) \in S_{k}}\left|I_{B}(x, y)-m_{k B}(x, y)\right|^{2}}
\end{array}\right.
$$


where $I$ is the input image and $M$ is the background image. $R, G$ and $B$ are the color components red, green and blue respectively of the pixel. The meaning of $m_{k}$ and $\sigma_{k}$ are mean value and standard deviation of each road area $S_{k}$, which is the predefined road area, and the meaning of $N_{S k}$ is total pixel number in $S_{k}$. The result of using (5) to the input image is shown in Fig. 5.

In fact, most of the road is near the color of dark gray. Although it is usually changing, it is still in a certain range. For RGB color model, a pixel is said to be gray with the property: $V_{r} \cong V_{g} \cong V_{b}$, so a pixel can be defined as a foreground pixel if one of its R,G, and B pixel values differs significantly.

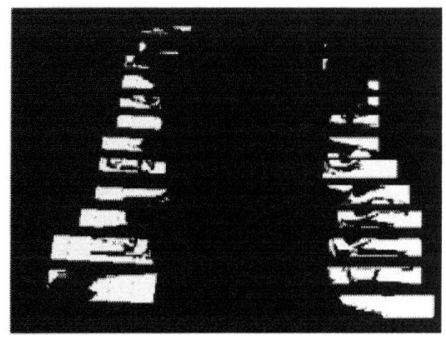

Fig. 5. The foreground image.

\section{Shadow detection and deduction}

In this section, the error from shadow will be deducted from the foreground image using chrominance information. After using foreground extraction, there would be some error which comes from the shadow of trees beside the parking space. The error comes from failing to distinguish between cars themselves and shadow. Nevertheless, drivers like to park their car in the shadow of trees because it is cooler. The shadow will make the color of parking space changeable, and it will increase the ratio of error. In fact, because it is just caused by the quantity of sunlight, most of pixel values are still within a range. When is shadowed, the degree of decrease of each RGB color space is in proportation. In other words, objects points, like cars, typically have an inter-frame difference greater than a threshold and it is not unusual that the ratio between shadows and reference image. Therefore, the foreground image extracted by (5) should be further modified by

$$
P^{\prime}(x, y)=\left\{\begin{array}{l}
\quad \text { if } \sqrt{\left(r_{R}-r_{G}\right)^{2}+\left(r_{G}-r_{B}\right)^{2}+\left(r_{B}-r_{R}\right)^{2}}<\eta \sqrt{\bar{f} / f_{0}} \\
0, \\
\text { and } \alpha \leq \frac{I_{f}(x, y)}{M_{f}(x, y)} \leq \beta \\
\text { 1,otherwise }
\end{array},\right.
$$

where $f$ is intensity (gray scale ) value, the same as (4). And $\bar{f}$ is the mean gray level value of input image. Parameters $\alpha$ and $\beta$ take into account how strong the light source is w.r.t the reflectance and irradiance of the objects. Thus, the stronger the sunlight (or the more the mean intensity $\bar{f}$ is), the lower $\alpha$ and higher $\beta$ should be chosen. In our system, $\eta=0.25$ and $f_{0}=145$ are empirical values. The value of $\left(r_{R}, r_{G}, r_{B}\right)$ will be obtained by

$$
\left\{\begin{array}{l}
r_{R}=I_{R}(x, y) /\left(M_{R}(x, y)+1\right) \\
r_{G}=I_{G}(x, y) /\left(M_{G}(x, y)+1\right) \\
r_{B}=I_{B}(x, y) /\left(M_{B}(x, y)+1\right)
\end{array}\right.
$$

The meaning of (7) is to find out whether the deviation of each pixel comes form shadow and remove them, and it is changeable because of the parameter $\bar{f}$.

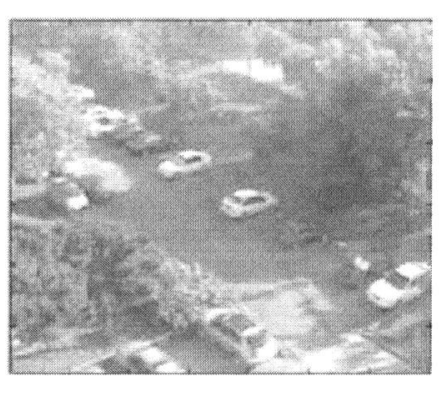

(a)

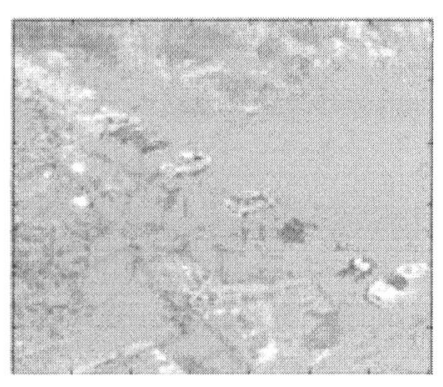

(b)
Fig. 6. (a) The original image. (b) Image after shadow detection: the pink pixels are those classified as shadow, and we can sec color of cars remains.

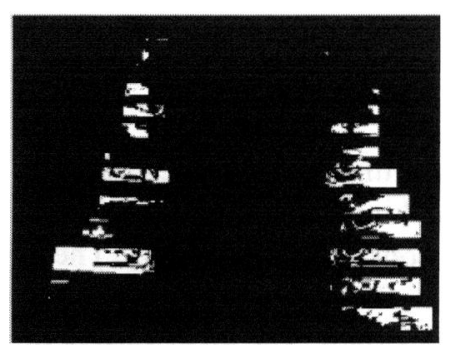

Fig. 7. The modified image.

Using RGB information increases the accuracy of the system. Using (7) doesn't define whether it is under shadow clearly. However, it is useful to distinguish the deviation from shadow and from cars themselves. Because that kind of deviation is just caused by illumination, most of the input images and their background images are still in a range of the similar chrominance. For each parking space, we can determine whether the stalls are available by

$$
R_{k}=\left\{\begin{array}{l}
1, \text { if } \rho>\tau_{p} \\
0, \text { otherwise }
\end{array}\right.
$$



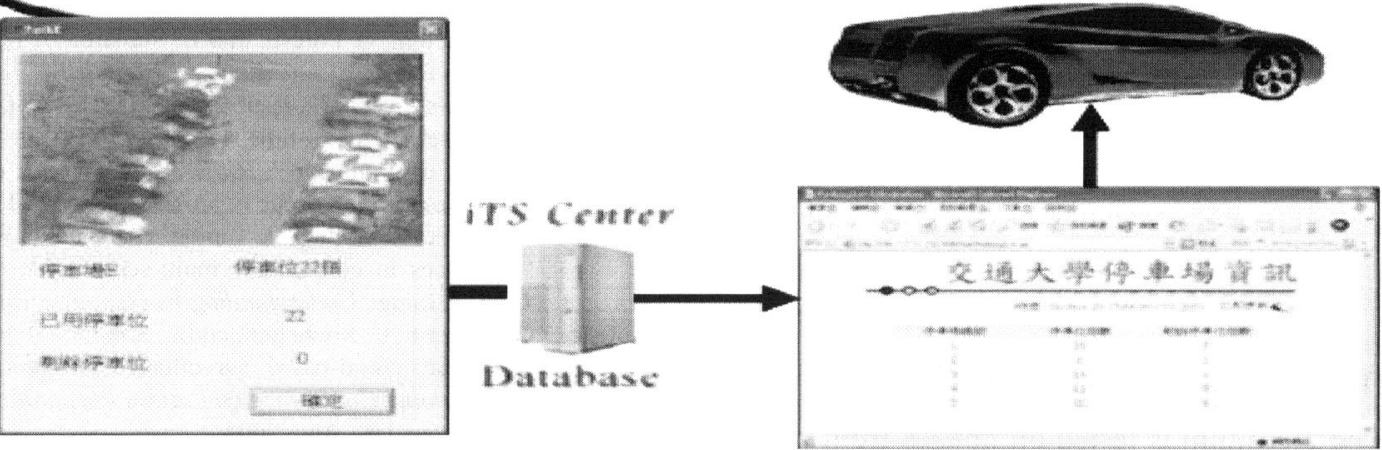

Fig. 8. Drivers can get real-time parking lot information by wireless communication device.

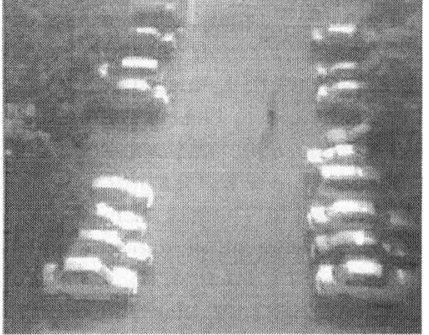

(a)

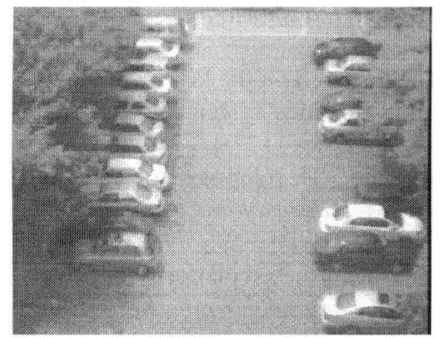

(c)

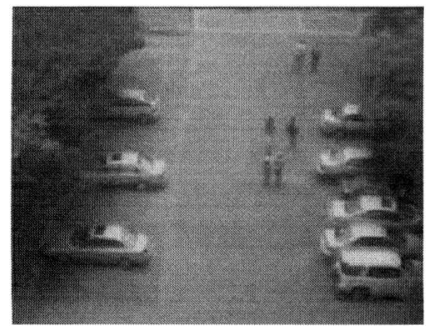

(i)

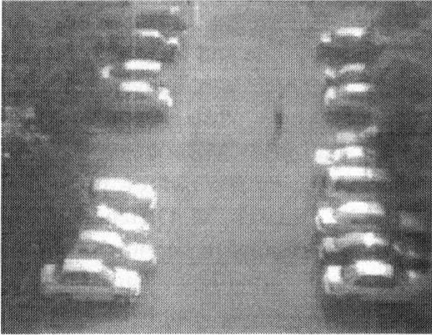

(b)

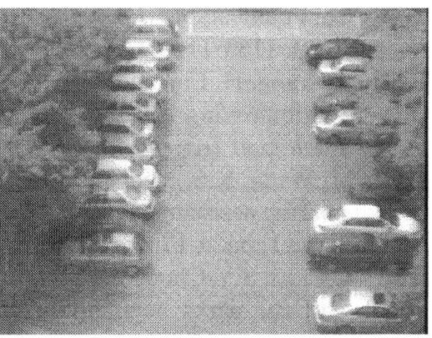

(f)

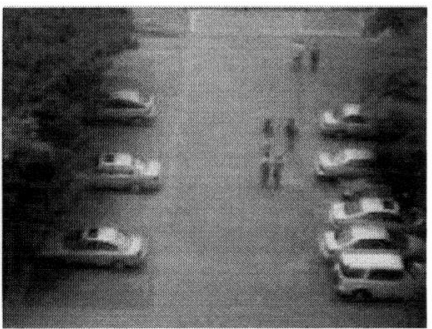

(j)

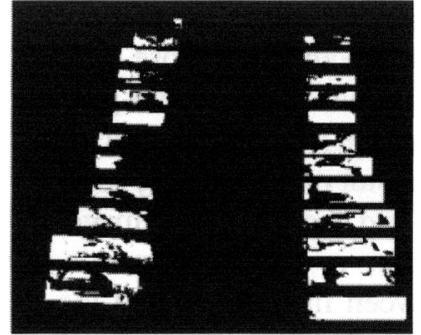

(c)

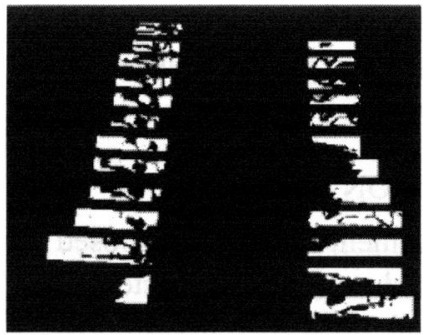

(g)

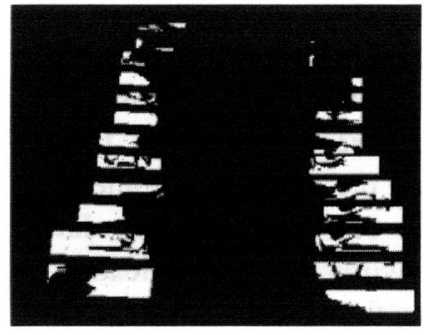

(k)

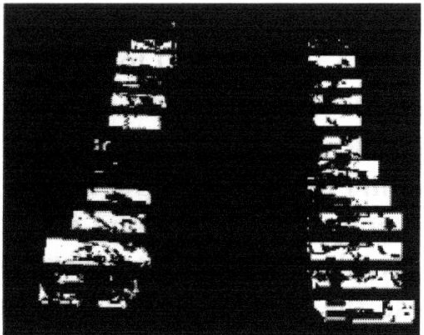

(d)

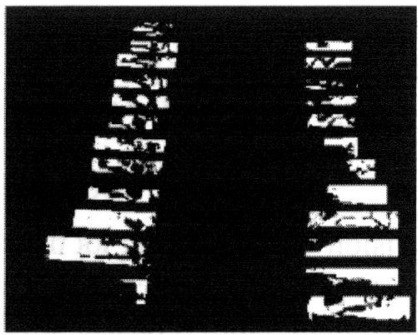

(h)

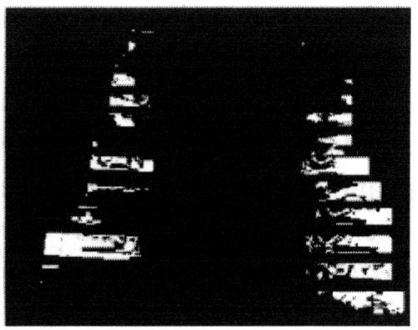

(1)

Fig. 9. (a)-(d) Experimental results of sunny day,(e)-(h) Experimental results of common weather. (i)-(1) Experimental results of cloudy day. Images from left to right are input image, result of performing color consistency, the foreground image, and the modified image, respectively.

The value of $\rho$ will be obtained by

$$
\rho=N_{p_{k}^{\prime}} / N_{B_{k}} \text {, }
$$

where $N_{B_{k}}$ is the total number of pixels which are in the parking space $B_{k}, N_{P_{k}}$, is the total number of pixels whose value equals to 1 in $B_{k}$, and $R_{k}$ is the final decision of each parking space. Parking space $B_{k}$ is classified as an occupied space if $\rho$ is sufficient large, in which case, $R_{k}$ is set to 1 . Otherwise, it is classified as a space available and $R_{k}$ is set to 0 . The choice of the parameter $\tau_{p}$ is less straight forward and, for now, is done empirically. 
At last, the vision-based parking lot management system calculates $R_{k}$ and integrates all information. Then, it sends information, which includes counting the number of parked vehicles, monitoring the changes of the parked vehicles over the time, identifying the stalls available to database of ITS center via internet and providing a user interface where drivers can get real-time parking lot information by wireless communication device. A user interface in this system is shown as Fig. 8.

\section{EXPERIMENTAL RESULTS}

The proposed algorithm is tested on images from cameras. All experimental images are 24-bit color images of size $352 \times 288$. Fig. 9 shows some images which are under different weather condition: (1) common weather whose gray level value is from 100 to 130 , (2) a cloudy day whose gray level value is from 70 to 120 , and (3) a sunny day whose gray level value is from 130 to 170 . The images from left to right, as shown in Figure8, are input image, result of performing color consistency, the foreground image, and the modified image, respectively. Sometimes errors occur, in our experiments, because the horizontal distance between the camera and the vehicle is too long. That is, an angle of elevation from vehicle to the camera is too little that it tends to be sheltered by other vehicles. However, experimental result indicates that this system is considerably precise and robust if the cameras are set high enough and near the parking lot.

\section{CONCLUSION}

A vision-based parking lot management system is proposed in this paper. A preprocessing procedure (color consistency) can maintain color consistency and raise stability of this system. Then, generating an adaptive parking lot background model based on color and predefined model enables this system suitable in most cases. Shadow detection method increases system accuracy. The vision-based method makes it possible to manage large area by just several cameras. It is cheap and easy-installed because of the simple equipment. However, drivers can get useful real-time parking lot information from this system by wireless communication device. It is an interesting topic in the future work to integrate this parking management system with other systems, such as traffic sign control system and traffic flow analysis system and it will improve the traffic greatly.

\section{REFERENCES}

[1] X. Wang and A. Hanson, "Parking lot analysis and visualization from aerial image," Proc. 4th IEEE Workshop on Applications of Computer Vision, pp.36-41, Oct. 1998.

[2] R. Collins, A. Lipton, H. Fujiyoshi, and T. Kanade, "Algorithms for cooperative multisensor surveillance," Proc. IEEE, vol. 89, Issuc 10, pp. 1456-1477, Oct. 2001.
[3] S. Dockstader and A. Tekalp, "Multiple camera tracking of interacting and occluded human motion," Proc. IEEE, vol. 89, Issue 10, pp. 1441-1455, Oct. 2001

[4] T. Matsuyama and N. Ukita, "Real-time multitarget tracking by a cooperative distributed vision system," Proc. IEEE, vol. 90, Issue 7, pp. 1136-1150, Jul. 2001.

[5] C. Micheloni, G. Foresti, and L. Snidaro, "A network of co-operative cameras for visual surveillance," Proc. IEE, Vision, Image, and Signal Processing, vol. 152, Issue 2, pp. 205-212, Apr. 2005.

[6] G. L. Foresti, "A real-time system for video surveillance of unattended outdoor environments", IEEE Trans. Circuits and Systems for Video Technology, vol. 8, Issue 6, pp. 697-704, Oct. 1998.

[7] T. Kanade, R. Collins, A. Lipton, P. Anandan, P. Burt, and L. Wixson, "Advances in cooperative multi-sensor video surveillance," Proc. DARPA Image Understanding Workshop, vol. 1, pp. 3-24, Nov. 1998.

[8] G.L. Foresti, C. Micheloni, and L. Snidaro, "Event classification for automatic visual-based surveillance of parking lots", Proc. 17th International Conference on Pattern Recognition, vol. 3, pp. 314-317, Cambridge, UK, Aug. 2004.

[9] Yuri Ivanov, Chris Stauffer, Aaron Bobick, and W. E. L. Grimson, "Video surveillance of interactions", Proc. 2nd IEEE Workshop on Visual Surveillance, pp.82-89, Jun. 1999.

[10] I. Haritaoglu, D. Harwood, and L.S. Davis, " $\mathrm{W}^{4}$ : real-time surveillance of people and their activities," IEEE Trans. Pattern Analysis and Machine Intelligence, vol. 22, Issue 8, pp. 809-830, Aug. 2000.

[11] U. Urfer, "Integration of systems and services in central monitoring stations (CMS)," Proc. 29th Annu. IEEE International Carnahan Conf. Security Technology, pp. 343-350, Sanderstead, UK, Oct. 1995.

[12] M. Braasch, M. DiBenedetto, S. Braasch, and R. Thomas, "LAAS operations in support of airport surface movement, guidance, control and surveillance: initial test result," Proc. IEEE Position Location and Navigation Symposium, San Diego, USA, pp. 82-89, Mar. 2000.

[13] H. Zhong, J. Xu, Y. Tu, Y. Hu, and J. Sun, "The research of parking guidance and information system based on dedicated short range communication," Proc. IEEE Intelligent Transportation Systems, vol. 2, pp. 1183-1186, Oct. 2003.

[14] Z. Yang, H. Liu, and X. Wang, "The research on the key technologies for improving efficiency of parking guidance system," Proc. IEEE Intelligent Transportation Systems, vol. 2, pp. 1177-1182, Oct. 2003.

[15] M. Wade, K.S. Yoon, and H. Hashimoto, "Development of advanced parking assistance system," IEEE Trans. Industrial Electronics, vol. 50, Issue 1, pp. 4-17, Feb. 2003.

[16] C.C. Li, S.Y. Chou, and S.W. Lin, "An agent-based platform for drivers and car parks negotiation," IEEE International Conf. Networking, Sensing and Control, vol. 2, pp. 1038-1043, Taipei, Taiwan, Mar. 2004. 